\title{
Using a New Auxiliary Equation to Construct Abundant Solutions for Nonlinear Evolution Equations
}

\author{
Yifan Liu' ${ }^{1}$ Guojiang $\mathbf{W u}^{2^{*}}$ \\ ${ }^{1}$ United Christian Academy, Civic Center Drive, Rancho Cucamonga, USA \\ ${ }^{2}$ Institute of Plasma Physics, Hefei Institutes of Physical Science, Chinese Academy of Sciences, Hefei, China \\ Email: *gjwu@ipp.ac.cn
}

How to cite this paper: Liu, Y.F. and $\mathrm{Wu}$, G.J. (2021) Using a New Auxiliary Equation to Construct Abundant Solutions for Nonlinear Evolution Equations. Journal of Applied Mathematics and Physics, 9, 31553164.

https://doi.org/10.4236/jamp.2021.912206

Received: November 24, 2021

Accepted: December 25, 2021

Published: December 28, 2021

Copyright $\odot 2021$ by author(s) and Scientific Research Publishing Inc. This work is licensed under the Creative Commons Attribution International License (CC BY 4.0).

http://creativecommons.org/licenses/by/4.0/

\begin{abstract}
In this paper, a new auxiliary equation method is proposed. Combined with the mapping method, abundant periodic wave solutions for generalized Klein-Gordon equation and Benjamin equation are obtained. They are new types of periodic wave solutions which are rarely found in previous studies. As $m \rightarrow 0$ and $m \rightarrow 1$, some new types of trigonometric solutions and solitary solutions are also obtained correspondingly. This method is promising for constructing abundant periodic wave solutions and solitary solutions of nonlinear evolution equations (NLEEs) in mathematical physics.
\end{abstract}

\section{Keywords}

Auxiliary Equation Method, Nonlinear Evolution Equations, Periodic Wave Solutions, Mapping Method, Solitary Wave Solutions

\section{Introduction}

NLEEs are widely used to describe complex phenomena in natural and social sciences. Many well-known models have been developed to illustrate the dynamics of nonlinear waves in the field of modern science and engineering, such as the Kortewegde Vries (KdV) [1] equation, KDV Burgers equation [2] [3], modified KDV (mKdV) equation [4], modified KDV Kadomtsev Petviashvili (mKdVKP) equation [5], and so on. More and more attention is focused on these nonlinear problems, and much nonlinear identification research can eventually be classified as NLEEs. Therefore, how to obtain their exact solutions is very important for the related nonlinear science research, and this has always been an important issue in the research of mathematics and physics [6]-[11]. Significant 
advancement has been produced in recent years and many strong and effective methods have been developed to obtain accurate solutions of NLEEs. For example, homogeneous balance method [12], algebraic method [13], the sine-cosine method [14], tanh-sech method and the extended tanh-coth method [15] [16], F-expansion method [17] [18], Exp-function method [19], Jacobi elliptic function expansion method [20] [21], the modified extended mapping method [22] [23] [24], auxiliary equation method [25] [26] [27], and so on. Based on previous original methods, the auxiliary equation method constructs the exact solution of ELEEs by introducing auxiliary equations. The application of good auxiliary equations can obtain a large number of new exact solutions of ELEEs. Therefore, finding appropriate auxiliary equations is of great significance to enrich the solution of NLEEs. In this paper, a new auxiliary equation is developed to construct new types of periodic wave solutions of NLEEs, which has not been proposed in previous work. With the cooperation of the previous extended mapping method, many new results are obtained.

\section{Method}

The following $(1+1)$-dimensional NLEE is considered

$$
N\left(u, u_{t}, u_{x}, u_{t t}, u_{x t}, u_{x x}, \cdots\right)=0
$$

Suppose Equation (1) has the following traveling wave solution

$$
u(x, t)=u(\xi), \quad \xi=x-\omega t
$$

where $\omega$ is a pending wave parameter. Substitute Equation (2) into Equation (1), and Equation (1) becomes the following ordinary differential equation

$$
N\left(u, u^{\prime}, u^{\prime \prime}, \cdots\right)=0
$$

where $u^{\prime}$ means $\mathrm{d} u / \mathrm{d} \xi$. Suppose Equation (3) has the following formal solution

$$
u(\xi)=\frac{\sum_{i=0}^{n} a_{i} f^{i}(\xi)}{f^{2}(\xi)+v}
$$

where $a_{i}$ and $v$ are constants to be determined later. The positive integer $n$ can be obtained by controlling the homogeneous balance between the governing nonlinear term and the highest order derivative of $u(\xi)$ in Equation (3). $f(\xi)$ is determined by the following auxiliary equation:

$$
f^{\prime}(\xi)=p f^{4}(\xi)+q f^{2}(\xi)+r
$$

where $p, q, r$ are parameters to be selected. In order to construct different types of periodic wave solutions, different $p, q, r$ are selected to determine the different Jacobi elliptic function solutions of Equation (5). Furthermore, these solutions include hyperbolic function solutions when $m \rightarrow 1$ and trigonometric function solutions when $m \rightarrow 0$. By using the mapping in Ref. [25], Equation (5) has the Jacobi elliptic function solutions as Table 1.

Where $i^{2}=-1$. Substituting Equation (4) and Equation (5) into (3), and setting the coefficients of $f^{i}(\xi) f^{\prime}(\xi)$ to zero yields a set of algebraic equations 
Table 1. The mapping of Jacobi elliptic function for Equation (5).

\begin{tabular}{|c|c|c|c|}
\hline$f(\xi)$ & $p$ & $q$ & $r$ \\
\hline$s n \xi, c d \xi=c n \xi / d n \xi$ & $m^{2}$ & $-\left(1+m^{2}\right)$ & 1 \\
\hline$c n \xi$ & $-m^{2}$ & $-1+2 m^{2}$ & $1-m^{2}$ \\
\hline$d n \xi$ & -1 & $2-m^{2}$ & $-1+m^{2}$ \\
\hline $\begin{array}{c}n s \xi=\frac{1}{s n \xi}, \\
d c \xi=d n \xi / c n \xi\end{array}$ & 1 & $-\left(1+m^{2}\right)$ & $m^{2}$ \\
\hline$n c \xi=1 / c n \xi$ & $1-m^{2}$ & $-1+2 m^{2}$ & $-m^{2}$ \\
\hline$n d \xi=1 / d n \xi$ & $-1+m^{2}$ & $2-m^{2}$ & -1 \\
\hline$c s \xi=c n \xi / s n \xi$ & 1 & $2-m^{2}$ & $1-m^{2}$ \\
\hline$s c \xi=s n \xi / c n \xi$ & $1-m^{2}$ & $2-m^{2}$ & 1 \\
\hline$s d \xi=s n \xi / d n \xi$ & $m^{2}\left(-1+m^{2}\right)$ & $-1+2 m^{2}$ & 1 \\
\hline$d s \xi=d n \xi / s n \xi$ & 1 & $-1+2 m^{2}$ & $m^{2}\left(-1+m^{2}\right)$ \\
\hline$m c n \xi \pm d n \xi$ & $-1 / 4$ & $\left(1+m^{2}\right) / 2$ & $-\left(1-m^{2}\right)^{2} / 4$ \\
\hline $\begin{array}{c}n s \xi \pm c s \xi \\
c n \xi /\left(\sqrt{1-m^{2}} s n \xi \pm d n \xi\right) \\
m s n \xi \pm i d n \xi \\
s n \xi /(1 \pm c n \xi)\end{array}$ & $1 / 4$ & $\left(1-2 m^{2}\right) / 2$ & $1 / 4$ \\
\hline$n c \xi \pm s c \xi, c n \xi /(1 \pm s n \xi)$ & $\left(1-m^{2}\right) / 4$ & $\left(1+m^{2}\right) / 2$ & $\left(1-m^{2}\right) / 4$ \\
\hline$n s \xi \pm d s \xi$ & $1 / 4$ & $\left(-2+m^{2}\right) / 2$ & $m^{4} / 4$ \\
\hline $\begin{array}{c}\operatorname{sn} \xi \pm i c n \xi \\
d n \xi /\left(\sqrt{m^{2}-1} s n \xi \pm c n \xi\right)\end{array}$ & $\mathrm{m}^{2} / 4$ & $\left(-2+m^{2}\right) / 2$ & $m^{2} / 4$ \\
\hline$d n \xi /\left(\sqrt{\frac{m^{2}-1}{m^{2}}} \pm c n \xi\right)$ & $\frac{1}{4 m^{2}}$ & $\left(1-2 m^{2}\right) / 2$ & $m^{2} / 4$ \\
\hline$s n \xi /(1 \pm d n \xi)$ & $m^{4} / 4$ & $\left(-2+m^{2}\right) / 2$ & $1 / 4$ \\
\hline$d n \xi /(1 \pm m s n \xi)$ & $\left(-1+m^{2}\right) / 4$ & $\left(1+m^{2}\right) / 2$ & $\left(-1+m^{2}\right) / 4$ \\
\hline$s n \xi /(c n \xi \pm d n \xi)$ & $\left(1-m^{2}\right)^{2} / 4$ & $\left(1+m^{2}\right) / 2$ & $1 / 4$ \\
\hline$c n \xi /\left(\sqrt{1-m^{2}} \pm d n \xi\right)$ & $m^{4} / 4$ & $\left(-2+m^{2}\right) / 2$ & $1 / 4$ \\
\hline
\end{tabular}

for $a_{i}$ and $v$. Solving the algebraic equations, $a_{i}$ and $v$ can be obtained expressed by $p, q, r$. Substituting these solutions into Equation (4) and using the mapping in Table 1, the new type of periodic wave solutions of Equation (3) can be obtained.

\section{Application of the Method}

\subsection{The Generalized Klein-Gordon Equation}

The following generalized Klein-Gordon equation [28] is considered 


$$
u_{t t}+\alpha u_{x x}+\beta u+\gamma u^{3}=0
$$

where $\alpha, \beta, \gamma$ are constants. Substituting the traveling wave solution Equation (2) into Equation (6) yields

$$
\left(\omega^{2}+\alpha\right) u^{\prime \prime}+\beta u+\gamma u^{3}=0
$$

By controlling the homogeneous balance between $u^{\prime \prime}$ and $u^{3}$ in Equation (7), $n=1$ can be obtained. So the solution of Equation (7) can be expressed as

$$
u(\xi)=\frac{a_{0}+a_{1} f(\xi)}{f^{2}(\xi)+v}
$$

Substituting Equation (8) into Equation (7) and use Equation (5) to yield a set of algebraic equations for $a_{0}, a_{1}$, and $v$. Solving the algebraic equations, $a_{0}, a_{1}$, and $v$ can be obtained as follows

$$
\begin{aligned}
& a_{0}=0, a_{1}= \pm \sqrt{\frac{-2 \beta v-\left(2 r-6 v q+2 v^{2} p\right)\left(\omega^{2}+\alpha\right)}{\gamma}}, \\
& v=\frac{6 r\left(\omega^{2}+\alpha\right)}{q\left(\omega^{2}+\alpha\right)+\beta}, \omega= \pm \sqrt{-\alpha+\frac{\beta}{-q \pm \sqrt{p r}}}
\end{aligned}
$$

By selecting different values of $p, q$ and $r$, the new type of periodic solutions of generalized Klein-Gordon equation can be obtained, and these solutions are rarely reported in other documents. Such as, if $p=m^{2}, q=-\left(1+m^{2}\right)$ and $r=1, f(\xi)=s n \xi$ and $f(\xi)=c d \xi$, the generalized Klein-Gordon equation has the following formal periodic solutions

$$
\begin{gathered}
u_{11}(\xi)= \pm \frac{\sqrt{\frac{-2 \beta v-\left(2+6\left(1+m^{2}\right) v+2 m^{2} v^{2}\right)\left(\omega^{2}+\alpha\right)}{\gamma}} s n \xi}{s n^{2} \xi+v} \\
u_{21}(\xi)= \pm \frac{\sqrt{\frac{-2 \beta v-\left(2+6\left(1+m^{2}\right) v+2 m^{2} v^{2}\right)\left(\omega^{2}+\alpha\right)}{\gamma} c d \xi}}{c d^{2} \xi+v} \\
\text { where } v=\frac{\sqrt{\frac{-2 \beta v-\left(2+6\left(1+m^{2}\right) v+2 m^{2} v^{2}\right)\left(\omega^{2}+\alpha\right)}{\gamma} c n \xi d n \xi}}{-\left(1+m^{2} \xi\left(\omega^{2} \xi+v d n^{2} \xi\right.\right.} \quad \text { (10) }
\end{gathered}
$$
$m \rightarrow 0$, it has the following new type of trigonometric solutions

$$
\begin{aligned}
& u_{12}(\xi)= \pm \frac{\sqrt{\frac{-2 \beta v-(2+6 v)\left(\omega^{2}+\alpha\right)}{\gamma}} \sin \xi}{\sin ^{2} \xi+v} \\
& u_{22}(\xi)= \pm \frac{\sqrt{\frac{-2 \beta v-(2+6 v)\left(\omega^{2}+\alpha\right)}{\gamma}} \cos \xi}{\cos ^{2} \xi+v}
\end{aligned}
$$


where $v=\frac{6\left(\omega^{2}+\alpha\right)}{-\left(\omega^{2}+\alpha\right)+\beta}, \xi=x-\omega t, \omega= \pm \sqrt{-\alpha+\beta}$. As $m \rightarrow 1$, it has the following new type of hyperbolic solutions

$$
u_{13}(\xi)= \pm \frac{\sqrt{\frac{-2 \beta v-\left(2+12 v+2 v^{2}\right)\left(\omega^{2}+\alpha\right)}{\gamma}} \tanh \xi}{\tanh ^{2} \xi+v}
$$

where $v=\frac{6\left(\omega^{2}+\alpha\right)}{-2\left(\omega^{2}+\alpha\right)+\beta}, \quad \xi=x-\omega t, \omega= \pm \sqrt{-\alpha+\frac{\beta}{2 \pm 1}}$.

$$
\text { If } p=m^{2} / 4, q=\left(-2+m^{2}\right) / 2 \text { and } r=1 / 4, \quad f(\xi)=s n \xi /(1 \pm d n \xi)
$$

$$
u_{31}(\xi)= \pm \frac{\sqrt{\frac{-2 \beta v-\left(\frac{1}{2}-3\left(-2+m^{2}\right) v+m^{2} v^{2} / 2\right)\left(\omega^{2}+\alpha\right)}{\gamma} \frac{s n \xi}{1 \pm d n \xi}}}{\frac{s n^{2} \xi}{(1 \pm d n \xi)^{2}}+v}
$$

where $v=\frac{3\left(\omega^{2}+\alpha\right) / 2}{\left(-2+m^{2}\right)\left(\omega^{2}+\alpha\right) / 2+\beta}, \quad \xi=x-\omega t$,

$\omega= \pm \sqrt{-\alpha+\frac{\beta}{-\left(-2+m^{2}\right) \pm m / 4}}$. As $m \rightarrow 0$, its trigonometric solution is the same as Equation (12). As $m \rightarrow 1$, it has the following new type of hyperbolic solutions

$$
u_{32}(\xi)= \pm \frac{\sqrt{\frac{-2 \beta v-\left(\frac{1}{2}+3 v+v^{2} / 2\right)\left(\omega^{2}+\alpha\right)}{\gamma} \frac{\tanh \xi}{1 \pm \operatorname{sech} \xi}}}{\frac{\tanh ^{2} \xi}{(1 \pm \operatorname{sech} \xi)^{2}}+v}
$$

where $v=\frac{3\left(\omega^{2}+\alpha\right) / 2}{-\left(\omega^{2}+\alpha\right) / 2+\beta}, \quad \xi=x-\omega t, \omega= \pm \sqrt{-\alpha+\frac{\beta}{1 \pm 1 / 4}}$.

The generalized Klein-Gordon equation still has other forms of solutions according to Equations (5), (8) and (9) and Table 1, limited to space, we will not give examples one by one.

\subsection{Benjamin Equation}

The following Benjamin equation is considered [29]

$$
u_{t t}+\alpha\left(u^{2}\right)_{x x}+\beta u_{x x x}=0
$$

where $\alpha, \beta$ are constants. The traveling wave Equation (2) is substituted into Equation (17) and integrated twice, and then the integration constant is set to zero to obtain

$$
\omega^{2} u+\alpha u^{2}+\beta u^{\prime \prime}=0
$$


By homogeneous balance, the solutions of Equation (17) can be expressed as

$$
u(\xi)=\frac{a_{0}+a_{1} f(\xi)+a_{2} f^{2}(\xi)}{f^{2}(\xi)+v}
$$

Substituting Equation (19) into Equation (18) and use (5) to yield a set of algebraic equations for $a_{0}, a_{1}, a_{2}$ and $v$. Solving the algebraic equations, $a_{0}, a_{1}, a_{2}$ and $v$ can be obtained as follows

$$
\begin{aligned}
& a_{0}=\frac{32 \beta q^{2}-4\left(\omega^{2}+4 \beta p\right) \sqrt{\beta q \pm\left(-p r \beta^{2}+\frac{\omega^{4}}{16}\right)}}{16 \alpha \beta p}, a_{1}=0, \\
& a_{2}=\frac{-\left(4 \beta q+\omega^{2}\right)+6\left(\beta q \pm \sqrt{-p r \beta^{2}+\frac{\omega^{4}}{16}}\right)}{2 \alpha}, \\
& v=\frac{\beta q \pm \sqrt{-p r \beta^{2}+\frac{\omega^{4}}{16}}, \omega= \pm\left(16 \beta q^{2}-48 p r \beta^{2}\right)^{1 / 4}}{2 \beta p},
\end{aligned}
$$

By selecting different values of $p, q$ and $r$, the new type of periodic solutions of Benjamin equation can be obtained, and these solutions are rarely reported in other documents. Such as, if $p=-m^{2}, q=-1+2 m^{2}$ and $r=-\left(1+m^{2}\right)$,

$f(\xi)=c n \xi$, the Benjamin equation has the following formal solutions

$$
\begin{aligned}
& u_{11}(\xi)= \frac{32 \beta\left(-1+2 m^{2}\right)^{2}-\left[-4 \omega^{2}+4 \beta\left(-1+2 m^{2}\right)\right] \sqrt{\beta\left(-1+2 m^{2}\right) \pm\left[-m^{2}\left(1+m^{2}\right) \beta^{2}+\frac{\omega^{4}}{16}\right]}}{c n^{2}(\xi)+\frac{\beta\left(-1+2 m^{2}\right) \pm \sqrt{-m^{2}\left(1+m^{2}\right) \beta^{2}+\frac{\omega^{4}}{16}}}{2 \beta m^{2}}} \\
&-\frac{-\left[\beta\left(-1+2 m^{2}\right)+\omega^{2}\right]+6\left[\beta \pm \sqrt{-m^{2}\left(1+m^{2}\right) \beta^{2}+\frac{\omega^{4}}{16}}\right]}{2 \alpha} c n^{2}(\xi) \\
& c n^{2}(\xi)+\frac{\beta\left(-1+2 m^{2}\right) \pm \sqrt{-m^{2}\left(1+m^{2}\right) \beta^{2}+\frac{\omega^{4}}{16}}}{2 \beta m^{2}}
\end{aligned}
$$

where $\xi=x-\omega t, \omega= \pm\left[16 \beta\left(-1+2 m^{2}\right)^{2}-48 m^{2}\left(1+m^{2}\right) \beta^{2}\right]^{1 / 4}$. The trigonometric solution does not exist in this type of Jacobi elliptic function solution. As $m \rightarrow 1$, it has the following new type of hyperbolic solution as

$$
u_{12}(\xi)=\frac{32 \beta+\frac{-4 \omega^{2}+4 \beta \sqrt{\beta \pm 2 \beta^{2}+\frac{\omega^{4}}{16}}}{16 \alpha \beta}-\frac{-\left(4 \beta+\omega^{2}\right)+6\left(\beta \pm \sqrt{-2 \beta^{2}+\frac{\omega^{4}}{16}}\right)}{2 \alpha} \tanh ^{2}(\xi)}{\tanh ^{2}(\xi)+\frac{-\beta \pm \sqrt{-2 \beta^{2}+\frac{\omega^{4}}{16}}}{2 \beta}}
$$


where $\xi=x-\omega t, \omega= \pm\left(16 \beta-96 \beta^{2}\right)^{1 / 4}$.

If $p=\left(-1+m^{2}\right) / 4, q=\left(1+m^{2}\right) / 2$ and $r=\left(-1+m^{2}\right) / 4$,

$f(\xi)=d n \xi /(1 \pm m s n \xi)$, the Benjamin equation has the following formal solutions

$$
\begin{aligned}
u_{21}(\xi)= & \frac{8 \beta\left(1+m^{2}\right)^{2}-4\left[\omega^{2}+\beta p\left(-1+m^{2}\right)\right] \sqrt{\beta \frac{\left(1+m^{2}\right)}{2} \pm\left[-\frac{\left(-1+m^{2}\right)^{2}}{16}\right] \beta^{2}+\frac{\omega^{4}}{16}}}{4 \alpha \beta\left(-1+m^{2}\right)} \\
-\frac{\frac{d n^{2}(\xi)}{(1 \pm m s n)^{2}}+\frac{2 \beta\left(1+m^{2}\right) \pm \sqrt{-\left(-1+m^{2}\right)^{2} \beta^{2}+\omega^{4}}}{2 \beta\left(-1+m^{2}\right)}}{2\left(1+m^{2}\right)-\omega^{2}+6\left[\beta \frac{\left(1+m^{2}\right)}{2} \pm \sqrt{\left.-\frac{\left(-1+m^{2}\right)^{2}}{16} \beta^{2}+\frac{\omega^{4}}{16}\right]} \frac{d n^{2}(\xi)}{(1 \pm m s n \xi)^{2}}\right.} & \frac{d n^{2}(\xi)}{(1 \pm m s n \xi)^{2}}+\frac{2 \beta\left(1+m^{2}\right) \pm \sqrt{-\left(-1+m^{2}\right)^{2} \beta^{2}+\omega^{4}}}{2 \beta\left(-1+m^{2}\right)}
\end{aligned}
$$

where $\xi=x-\omega t, \omega= \pm\left[4 \beta\left(1+m^{2}\right)^{2}-3\left(-1+m^{2}\right)^{2} \beta^{2}\right]^{1 / 4}$. The trigonometric solution and hyperbolic solution all do not exist in this type of Jacobi elliptic function solution.

If $p=\left(1-m^{2}\right)^{2} / 4, q=\left(1+m^{2}\right) / 2$ and $r=1 / 4, \quad f(\xi)=s n \xi /(c n \xi \pm d n \xi)$, the Benjamin equation has the following formal solutions

$$
\begin{aligned}
u_{31}(\xi)= & \frac{8 \beta\left(1+m^{2}\right)^{2}-4\left[\omega^{2}+\beta\left(1-m^{2}\right)^{2}\right] \sqrt{\beta \frac{\left(1+m^{2}\right)}{2} \pm\left[-\frac{\left(1-m^{2}\right)^{2}}{16} \beta^{2}+\frac{\omega^{4}}{16}\right]}}{4 \alpha \beta\left(1-m^{2}\right)^{2}} \\
& -\frac{\frac{s n^{2}(\xi)}{(c n \xi \pm d n \xi)^{2}}-\frac{2 \beta\left(1+m^{2}\right) \pm \sqrt{-\left(1-m^{2}\right)^{2} \beta^{2}+\omega^{4}}}{2 \beta\left(1-m^{2}\right)}}{\frac{-\left[\beta \left(\beta \frac{\left(1+m^{2}\right)}{2} \pm \sqrt{\left.\left.-\frac{\left(1-m^{2}\right)^{2}}{16} \beta^{2}+\frac{\omega^{4}}{16}\right)\right]} \frac{s n^{2}(\xi)}{(c n \xi \pm d n \xi)^{2}}\right.\right.}{2 \alpha}}
\end{aligned}
$$

where $\xi=x-\omega t, \omega= \pm\left[16 \beta q^{2}-3\left(1-m^{2}\right)^{2} \beta^{2}\right]^{1 / 4}$. The hyperbolic solution does not exist in this type of Jacobi elliptic function solution. As $m \rightarrow 0$, it has the following new type of trigonometric solution as 


$$
u_{32}(\xi)=\frac{\frac{2 \beta-4\left(\omega^{2}+\beta\right) \sqrt{8 \beta \pm\left(-\beta^{2}+\omega^{4}\right)}}{\alpha \beta}-\frac{-\left[6\left(2 \beta \pm \sqrt{-\beta^{2}+\omega^{4}}\right)\right]}{8 \alpha} \frac{\sin ^{2}(\xi)}{(\cos \xi \pm 1)^{2}}}{\frac{\sin ^{2}(\xi)}{(\cos \xi \pm 1)^{2}}-\frac{2 \beta \pm \sqrt{-\beta^{2}+\omega^{4}}}{2 \beta}}
$$

where $\xi=x-\omega t, \omega= \pm\left(4 \beta-3 \beta^{2}\right)^{1 / 4}$

There are still a large number of new types of periodic wave solutions for Benjamin equation, according to Equations (5), (8) and (9) and Table 1. Accordingly, these solutions may also have trigonometric function solutions and hyperbolic function solutions under the conditions of $m \rightarrow 0$ and $m \rightarrow 1$, of course, they may not exist. Limited to the scope, we will not give examples one by one

\section{Conclusion}

In this paper, with the use of a new auxiliary Equation (4) and the extended mapping method (Table 1), abundant new types of Jacobi elliptic function solutions for the generalized Klein-Gordon equation and Benjamin equation are constructed. Some new types of periodic wave solutions and solitary wave solutions have been obtained which have not been found in previous work. The obtained periodic wave solutions and solitary solutions imply that the corresponding periodic wave and solitary wave can be generated under certain conditions of phase space $(x, y)$ and time $t$. Our method is only to find new periodic solutions and solitary solutions of NLEEs mathematically. The experimental verification needs to design experiments in specific fields to verify the physical significance of our solutions, which we can't do in this paper. But, despite all this, this method is still promising for constructing abundant periodic wave solutions and solitary solutions and can serve as a useful guide for a broad class of nonlinear problems in the study of mathematics and physics.

\section{Conflicts of Interest}

The authors declare no conflicts of interest regarding the publication of this paper.

\section{References}

[1] Gardner, C.S., Greene, J.M., Kruskal, M.D. and Miura, R.M. (1967) Method for Solving Korteweg-Devries Equation. Physical Review Letters, 19, 1095-1097. https://doi.org/10.1103/PhysRevLett.19.1095

[2] Su, C.H. and Gardner, C.S. (1969) Korteweg-De Vries Equation and Generalizations. 3. Derivation of Korteweg-De Vries Equation and Burgers Equation. Journal of Mathematical Physics, 10, 536-539. https://doi.org/10.1063/1.1664873

[3] Li, Z.B. and Wang, M.L. (1993) Traveling-Wave Solutions to the 2-Dimensional Kdv Burgers-Equation. Journal of Physics A-Mathematical and General, 26, 6027-6031. https://doi.org/10.1088/0305-4470/26/21/039

[4] Ito, M. (1980) An Extension of Non-Linear Evolution-Equations of the K-DV 
(MK-DV) Type to Higher Orders. Journal of the Physical Society of Japan, 49, 771-778. https://doi.org/10.1143/JPSJ.49.771

[5] Wang, M.L. (1995) Solitary Wave Solutions for Variant Boussinesq Equations. Physics Letters A, 199, 169-172. https://doi.org/10.1016/0375-9601(95)00092-H

[6] Guo, H.D., Xia, T.C. and Hu, B.B. (2020) High-Order Lumps, High-Order Breathers and Hybrid Solutions for an Extended $(3+1)$-Dimensional Jimbo-Miwa Equation in Fluid Dynamics. Nonlinear Dynamics, 100, 601-614. https://doi.org/10.1007/s11071-020-05514-9

[7] Lan, Z.Z. and Guo, B.L. (2020) Nonlinear Waves Behaviors for a Coupled Generalized Nonlinear Schrodinger-Boussinesq System in a Homogeneous Magnetized Plasma. Nonlinear Dynamics, 100, 3771-3784. https://doi.org/10.1007/s11071-020-05716-1

[8] Biswas, A., Ekici, M., Sonmezoglu, A. and Belic, M.R. (2019) Solitons in Optical Fiber Bragg Gratings with Dispersive Reflectivity by Extended Trial Function Method. Optik, 182, 88-94. https://doi.org/10.1016/j.ijleo.2018.12.156

[9] Seadawy, A.R., Lu, D.C., Nasreen, N. and Nasreen, S. (2019) Structure of Optical Solitons of Resonant Schrodinger Equation with Quadratic Cubic Nonlinearity and Modulation Instability Analysis. Physica A, 534, Article ID: 122155. https://doi.org/10.1016/j.physa.2019.122155

[10] Abdoud, M.A., Owyed, S., Abdel-Aty, A., Raffan, B.M. and Abdel-Khalek, S. (2020) Optical Soliton Solutions for a Space-Time Fractional Perturbed Nonlinear Schrödinger Equation Arising in Quantum Physics. Results in Physics, 16, Article ID: 102895. https://doi.org/10.1016/j.rinp.2019.102895

[11] Peng, W.Q., Tian, S.F. and Zhang, T.T. (2019) Dynamics of the Soliton Waves, Breather Waves, and Rogue Waves to the Cylindrical Kadomtsev-Petviashvili Equation in Pair-Ion-Electron Plasma. Physics of Fluids, 31, Article ID: 102107. https://doi.org/10.1063/1.5116231

[12] Seadawy, A.R. (2016) Stability Analysis Solutions for Nonlinear Three-Dimensional Modified Korteweg-de Vries-Zakharov-Kuznetsov Equation in a Magnetized Electron-Positron Plasma. Physica A, 455, 44-51. https://doi.org/10.1016/j.physa.2016.02.061

[13] Arshad, M., Seadawy, A.R., Lu, D.C. and Wang, J. (2017) Travelling Wave Solutions of Drinfel'd-Sokolov-Wilson, Whitham-Broer-Kaup and (2+1)-Dimensional BroerKaup-Kupershmit Equations and Their Applications. Chinese Journal of Physics, 55, 780-797. https://doi.org/10.1016/j.cjph.2017.02.008

[14] Yan, C.T. (1996) A Simple Transformation for Nonlinear Waves. Physics Letters A, 224, 77-84. https://doi.org/10.1016/S0375-9601(96)00770-0

[15] Seadawy, A.R. (2014) Stability Analysis for Zakharov-Kuznetsov Equation of Weakly Nonlinear Ion-Acoustic Waves in a Plasma. Computers \& Mathematics with Applications, 67, 172-180. https://doi.org/10.1016/j.camwa.2013.11.001

[16] Seadawy, A.R. (2014) Stability Analysis for Two-Dimensional Ion-Acoustic Waves in Quantum Plasmas. Physics of Plasmas, 21, Article ID: 052107. https://doi.org/10.1063/1.4875987

[17] Liu, J.B. and Yang, K.Q. (2004) The Extended F-Expansion Method and Exact Solutions of Nonlinear PDEs. Chaos, Solitons \& Fractals, 22, 111-121. https://doi.org/10.1016/j.chaos.2003.12.069

[18] Zhang, S. (2007) Application of Exp-Function Method to a KdV Equation with Variable Coefficients. Physics Letters A, 365, 448-453.

https://doi.org/10.1016/j.physleta.2007.02.004 
[19] Shek, E.C. M. and Chow, K.W. (2008) The Discrete Modified Korteweg-de Vries Equation with Non-Vanishing Boundary Conditions: Interactions of Solitons. Chaos, Solitons \& Fractals, 36, 296-302. https://doi.org/10.1016/j.chaos.2006.06.036

[20] Liu, S.K., Fu, Z.T., Liu, S.D. and Zhao, Q. (2001) Jacobi Elliptic Function Expansion Method and Periodic Wave Solutions of Nonlinear Wave Equations. Physics Letters $A$, 289, 69-74. https://doi.org/10.1016/S0375-9601(01)00580-1

[21] Boateng, K., Yang, W.G., Yaro, D. and Otoo, M.E. (2020) Jacobi Elliptic Function Solutions and Traveling Wave Solutions of the $(2+1)$-Dimensional Gardner-KP Equation. Mathematical Methods in the Applied Sciences, 43, 3457-3472. https://doi.org/10.1002/mma.6131

[22] Li, H.M. (2002) Searching for the (3+1)-Dimensional Painleve Integrable Model and Its Solitary Wave Solution. Chinese Physics Letters, 19, 745-747.

https://doi.org/10.1088/0256-307X/19/6/301

[23] Yomba, E. (2004) On Exact Solutions of the Coupled Klein-Gordon-Schrodinger and the Complex Coupled KdV Equations Using Mapping Method. Chaos, Solitons \& Fractals, 21, 209-229. https://doi.org/10.1016/j.chaos.2003.10.028

[24] Li, H.M. (2005) New Exact Solutions of Nonlinear Gross-Pitaevskii Equation with Weak Bias Magnetic and Time-Dependent Laser Fields. Chinese Physics B, 14, 251-256.

[25] Wu, G., Han, J., Zhang, W. and Zhang, M. (2007) New Periodic Wave Solutions to Nonlinear Evolution Equations by the Extended Mapping Method. Physica D: Nonlinear Phenomena, 229, 116-122. https://doi.org/10.1016/j.physd.2007.03.015

[26] Sirendaoreji (2004) New Exact Travelling Wave Solutions for the Kawahara and Modified Kawahara Equations. Chaos, Solitons \& Fractals, 19, 147-150. https://doi.org/10.1016/S0960-0779(03)00102-4

[27] Tariq, K.U. H. and Seadawy, A.R. (2017) Bistable Bright-Dark Solitary Wave Solutions of the $(3+1)$-Dimensional Breaking Soliton, Boussinesq Equation with Dual Dispersion and Modified Korteweg-de Vries-Kadomtsev-Petviashvili Equations and Their Applications. Results in Physics, 7, 1143-1149. https://doi.org/10.1016/j.rinp.2017.03.001

[28] El-Wakil, S.A., Elgarayhi, A. and Elhanbaly, A. (2006) Exact Periodic Wave Solutions for Some Nonlinear Partial Differential Equations. Chaos, Solitons \& Fractals, 29, 1037-1044. https://doi.org/10.1016/j.chaos.2005.08.063

[29] Sirendaoreji and Taogetusang (2006) New Type of Exact Solitary Wave Solutions for Dispersive Long-Wave Equation and Benjamin Equation. Acta Physica Sinica, 55, 3246-3254. https://doi.org/10.7498/aps.55.3246 